\title{
Printing Ink Excipient
}

National Cancer Institute

\section{Source}

National Cancer Institute. Printing Ink Excipient. NCI Thesaurus. Code C42657.

The constituents of printing inks used to mark an ingested pharmaceutical dosage form. 